\title{
Cross-sectional associations of objectively assessed sleep duration with physical activity, BMI and television viewing in German primary school children
}

\author{
Susanne Kobel ${ }^{1 *}$ (D) Olivia Wartha ${ }^{1}$, Jens Dreyhaupt ${ }^{2}$, Sarah Kettner ${ }^{1}$ and Jürgen M. Steinacker ${ }^{3}$
}

\begin{abstract}
Background: The global incidence of overweight and obesity has increased dramatically among children and adolescents over the past decades. Insufficient sleep duration and physical inactivity are known risk factors for overweight and obesity in children. To engage children in a healthier lifestyle knowledge about associations of sleep duration and behavioural aspects in children are vital. Therefore, this study investigated the mentioned associations in German primary school children.
\end{abstract}

Methods: Data of 308 first and second graders (7.1 \pm 0.6 years) was used; children's anthropometric data were taken during a school visit. Children's physical activity (PA) and sleep duration were assessed objectively (Actiheart $\odot$, CamNtech Ltd., Cambridge, UK); children's daily television time and socio-demographic data were collected via parental questionnaire. Linear mixed-effects regression models as well as logistic regressions were used to determine associations of PA, television viewing, age, gender, BMI z-scores and socio-economic variables on sleep duration.

Results: In linear regression models young age and not having a migration background were significantly associated with long sleep duration $(p<0.001)$. In logistic regressions, long night time sleep $(\geq 10: 08 \mathrm{~h}$; compared to medium and short sleep duration) was significantly associated with not reaching the PA guideline (OR 0.60 [0.36;0. 99]), daily television viewing of less than one hour (OR 0.44 [0.24;0.80]), young age (OR 0.38 [0.21;067]), a high parental education level (OR $0.52[0.27 ; 0.99])$ and the lack of migration background (OR $0.21[0.10 ; 0.48])$. However, if controlling for age, gender, parental education level and migration background, reaching the PA guideline stayed no longer significantly associated with a tertiary sleep level.

Conclusions: Children in the highest sleep category showed a negative association with reaching the PA guideline and a positive association with daily television viewing. This therefore adds to previously primarily subjectively assessed associations of sleep and risk factors for obesity (related behaviours) with a detailed insight based on objective data. Hence, interventions trying to decrease children's BMI and television viewing should also aim at extending children's night-time sleep and inform parents about the importance of sufficient sleep during childhood.

Trial registration: DRKS-ID: DRKS00000494.

Keywords: MVPA, Body composition, TV watching, Accelerometer, Migration

\footnotetext{
* Correspondence: susanne.kobel@uni-ulm.de

${ }^{1}$ Division of Sports and Rehabilitation Medicine, Department of Internal

Medicine II, Ulm University Medical Centre, Frauensteige 6, Haus 58/33,

D-89075 Ulm, Germany

Full list of author information is available at the end of the article
}

(c) The Author(s). 2019 Open Access This article is distributed under the terms of the Creative Commons Attribution 4.0 International License (http://creativecommons.org/licenses/by/4.0/) which permits unrestricted use, distribution, and reproduction in any medium, provided you give appropriate credit to the original author(s) and the source, provide a link to the Creative Commons license, and indicate if changes were made. The Creative Commons Public Domain Dedication waiver (http://creativecommons.org/publicdomain/zero/1.0/) applies to the data made available in this article, unless otherwise stated. 


\section{Background}

The emergence of overweight and obesity has become a global health problem that no longer affects only wealthier industrialised countries, but is also increasingly spreading in developing countries [1]. The increase in body weight can lead to serious health consequences such as cardiovascular diseases [2], metabolic disorders, such as diabetes mellitus [3], psychological and motor developmental delays [4], and emotional stress [5].

The global incidence of overweight and obesity has also increased dramatically among children and adolescents over the past 30 years [6]. If this trend continues, experts predict $11 \%$ of overweight children worldwide in 2025 [7], already in 2016, over 41 million children under the age of five are estimated to be overweight [8]. Since children are dependent on their parent's behaviour, especially their nutrition and physical activity behaviour $[9,10]$, it is important to introduce children to a healthy lifestyle as early as possible in order to embed healthy habits into their daily life [11]. Reasons for children's energy unbalance are increased energy intake, often caused by the consumption of sugary drinks as well as too little physical activity and too high screen media use [12]. This trend is particularly evident in children starting school [13, 14].

In order to counteract this and since regular physical activity has shown to have numerous benefits to children's health $[15,16]$ the World Health Organisation advocates a daily amount of $60 \mathrm{~min}$ of moderate to vigorous physical activity (MVPA) for children and adolescents [17]. In spite of this, many children are not sufficiently active enough to benefit their health. Depending on assessment method, interpretation, sample and region, between $87 \%$ and $3-5 \%$ of European youth is considered sufficiently active [18]. In the US, nearly $50 \%$ of children fail to meet the minimum requirement for daily physical activity [19] and less than a third and a quarter of English boys and girls, respectively, between two and 15 years meet the recommended $60 \mathrm{~min}$ or more of MVPA a day [20]. In Germany, less than 20\% of primary school children are sufficiently active [21].

However, not only physical inactivity, but also insufficient sleep duration is a recognised risk factor for children's health [22-25], which importance grows from the time of school entry [26]. Current recommendations, considering children's overall well-being as well as cognitive, emotional and physical health, advocate nine to eleven hours of sleep per day for 6- to 13-year-olds [27]. Based on those recommendations, internationally, numerous studies investigated the influence of sleep on obesity and obesity-related behaviours in children $[28,29]$. In German as well as Chinese primary school children for instance, a daily sleep duration of $10 \mathrm{~h}$ or less was significantly associated to being overweight or obese [26, 28]. Similarly, boys who slept less than $10 \mathrm{~h}$ per night showed an increased risk of higher fasting blood glucose [29] and a large review found consistent associations between short sleep duration and more screen media use [30].

Further, there have been many attempts to find associations between children's night-time sleep duration and their physical activity behaviour during the day - especially considering the physical activity guideline of $60 \mathrm{~min}$ of moderate to vigorous physical activity (MVPA) daily [17] - although so far with inconsistent findings. Previous research has shown that children's sleep duration was positively [31], negatively [32, 33], or not at all [24, 34, 35] related to their physical activity behaviour on the next day. The reasons for these inconsistencies remain unclear; however, many of the studies examining sleep duration base their data on subjective assessments, such as questionnaires or proxy reports, which have known disadvantages and partially invalid for assessing sleep duration [36]. Especially in Germany, there is a lack of data on objectively assessed children's sleep duration and physical activity. Hence, this study investigated associations of objectively assessed sleep duration with physical activity behaviour, body composition and television viewing in German primary school children.

\section{Methods}

\section{Study population}

Baseline data of a sub-sample of 308 first and second graders of 32 primary schools $(7.1 \pm 0.6$ years) taking part in the so-called Baden-Württemberg study in south-west Germany [37], was used. Details on its study design and protocol as well as the recruiting process can be found elsewhere [38]. Parents provided their written informed consent to take part in the study and a separate consent for their children to wear a multi-sensor device assessing sleep and physical activity objectively for six consecutive days; children gave their verbal assent on the day of assessment. The study was approved by the university's ethics committee (application no. 126/10) and conducted in accordance with the declaration of Helsinki.

\section{Anthropometric measurements}

Children's anthropometric data (height $(\mathrm{cm})$ and body weight $(\mathrm{kg})$ ) were taken by trained staff using a stadiometer and calibrated electronic scales (Seca 213 and Seca 826, respectively, Seca Weighing and Measuring Systems, Hamburg, Germany). Subsequently, children's body mass index (BMI) was calculated $\left(\mathrm{kg} / \mathrm{m}^{2}\right)$ and converted to BMI z-scores [39].

\section{Sleep and physical activity assessment}

Children's physical activity and sleep duration were assessed objectively by a chest-worn multi-sensor device 
(Actiheart $\odot$, CamNtech Ltd., Cambridge, UK) which measures uniaxial acceleration in combination with heart rate. Validity of the device in children has been established previously [40]. Although this technique is primarily used to assess physical activity and sedentary behaviour, it has found increasing use in the field of sleep medicine in recent years $[41,42]$.

The device's recording interval was set to $15 \mathrm{~s}$ and participants wore it for six consecutive days and nights (for further details see [43]). To be included in the physical activity analyses, at least three days (including at least one weekend day) of valid data of more than $10 \mathrm{~h}$ were required (as recommended by Trost et al. [44]). First and last recording days were excluded from the analysis to antagonise a novelty factor on the first day, whereas the last day never showed $10 \mathrm{~h}$ of recording. In order to show valid sleep data, at least three nights, including at least one at the weekend were necessary (as recommended by the American Academy of Sleep Medicine [45]). Individual sleep duration was based on the children's individual heart rate variability in combination with assessed inactivity, which allows the determination of the exact time of falling asleep and awaking. Both of which was identified by two independent experts and subsequently calculated in hours per night-time sleep.

Children's physical activity levels were determined on the basis of energy expenditure (MET) predicted by Actiheart@'s captive software (Version 4.0.129), taking into account participant's age, height, body weight and gender in addition to the assessed heart rate and movement counts. Physical activity was then classified into sedentary (<1.5 MET), light (1.5-3 MET), moderate (> 3-6 MET), and vigorous (> $6 \mathrm{MET}$ ) as well as MVPA (> $3 \mathrm{MET}$ ) for each $15 \mathrm{~s}$ recording interval [46]. In order to determine whether participants met the WHO physical activity guideline of $60 \mathrm{~min}$ of MVPA every single day [17], the days with valid data were extrapolated to a full week, using a ratio of 5:2 for weekdays and weekend days.

\section{Behavioural and socio-demographic data}

Behavioural and socio-demographic data were collected using standardised and validated questions [47] in a parental questionnaire. Children's daily television time was assessed for weekdays as well as weekend days on a categorical level. A ratio of 5:2 was used to determine daily television time. Parental education was based on the highest school education of either one parent or the single parent. Further, children were classified as having a migration background if at least one parent was born abroad or the child was spoken to in foreign language during the first three years of life.

\section{Statistical analysis}

For logistic regressions, children's sleep duration in hours and minutes was dichotomised by a primary/secondary and tertiary sleep level (total sleep duration of all children was split in three parts of equal frequency); therefore, tertiary sleep level was defined as an average sleep duration of $10 \mathrm{~h}$ and $8 \mathrm{~min}$ or more (compared to primary sleep level of 9:45 h or less or a secondary sleep level of between 9:46 $\mathrm{h}$ and 10:07 h). For logistic regression analyses, physical activity (in minutes per day) was dichotomised by reaching the WHO physical activity guideline of $60 \mathrm{~min}$ of MVPA every single day or not. Daily television viewing was dichotomised by one hour or more (median split as well as German recommendations [48]). For logistic regression analyses, children's age was dichotomised at below seven years and seven years or more (median split). Socio-economic variables, such as parental education was dichotomised by primary/secondary and tertiary education level; i.e. having a high school degree or not; and children with at least one parent who was born abroad or were spoken to in foreign language during the first three years of life, were dichotomised as having a migration background.

Group differences between means were analysed with unpaired t-tests; linear regression and ANOVA were used to examine differences in BMI z-scores on the basis of children's sleep duration. Linear mixed-effects regression models were used to determine associations of physical activity, television viewing, age, gender, BMI z-scores and socio-economic variables such as parental education and migration background on sleep duration, controlling for school effects. Logistic regressions were calculated for physical activity and television viewing, controlling for age, parental education and migration background.

Descriptive statistics for continuous variables are displayed in mean values and standard deviations. Categorical variables are described with absolute and relative frequencies. Statistical analyses were performed using SPSS Statistics 25 (SPSS Inc., Chicago, IL, US) and SAS (SAS Institute, Cary, NC, US) with a significance level set to $\alpha<0.05$.

\section{Results}

\section{Sample characteristics}

There are no differences between the here analysed subsample $(n=308)$ and the overall sample of the Baden-Württemberg study $(n=1947)$ with regard to the parameters age, gender, BMI z-scores, parental education level and migration background. A summary of participant's anthropometric and socio-demographic characteristics as well as their physical activity level and television viewing is shown in Table 1. 
Table 1 Descriptive characteristics of total sample, children with short/medium sleep duration (primary and secondary sleep level; < $10 \mathrm{~h}, 8 \mathrm{~min}$ ) and children with long sleep duration (tertiary sleep level; $\geq 10 \mathrm{~h}, 8 \mathrm{~min}$ )

\begin{tabular}{|c|c|c|c|c|}
\hline & Missing values & Total sample $(n=308)$ & $\begin{array}{l}\text { Short/medium } \\
\text { Sleep Duration }(n=202)\end{array}$ & Long Sleep Duration $(n=106)$ \\
\hline Gender (male); n (\%) & 0 & $151(49.0)$ & $106(52.5)$ & $45(42.5)$ \\
\hline Age $($ years)*; $m \pm S D$ & 0 & $7.1 \pm 0.6$ & $7.2 \pm 0.6$ & $6.9 \pm 0.6$ \\
\hline Height $(\mathrm{cm}) ; \mathrm{m} \pm \mathrm{SD}$ & 1 & $123.6 \pm 6.1$ & $124.0 \pm 6.1$ & $122.7 \pm 6.0$ \\
\hline Weight $(\mathrm{kg})^{*} ; \mathrm{m} \pm \mathrm{SD}$ & 1 & $24.6 \pm 4.8$ & $25.0 \pm 4.8$ & $23.8 \pm 4.7$ \\
\hline BMI z-scores; $\mathrm{m} \pm \mathrm{SD}$ & 1 & $0.18 \pm 1.11$ & $0.25 \pm 1.11$ & $0.03 \pm 1.10$ \\
\hline Weight status & 13 & & & \\
\hline Overweight; n (\%) & 13 & $36(12.2)$ & $26(13.5)$ & $10(9.8)$ \\
\hline Obese; n (\%) & 13 & $10(3.4)$ & $6(3.1)$ & $4(3.9)$ \\
\hline Sleep duration (h:min/night)*; $m \pm S D$ & 0 & $9: 58(0: 29)$ & $9: 42(0: 19)$ & $10: 29(0: 18)$ \\
\hline MVPA Guideline reached*; n (\%) & 4 & $148(48.7)$ & $105(52.8)$ & $43(41.0)$ \\
\hline Daily MVPA (h:min/day)*,$m \pm S D$ & 2 & $2: 13(0: 58)$ & $2: 18(0: 59)$ & 2:04 (0:55) \\
\hline TV viewing of more than $1 \mathrm{~h} /$ day $^{*} ; \mathrm{n}(\%)$ & 41 & $150(56.2)$ & $108(63.2)$ & $42(43.8)$ \\
\hline Tertiary parental education level; n (\%) & 48 & $92(35.4)$ & $59(36.2)$ & $33(34.0)$ \\
\hline Migration background*; n (\%) & 41 & 71 (26.6) & $60(35.7)$ & $11(11.1)$ \\
\hline
\end{tabular}

Values are mean $(m) \pm$ SD or numbers $(n)$ and percentages (\%). Overweight and obese defined as per Cole et al. (2000); MVPA guideline reached = a minimum of 60 min of MVPA (moderate to vigorous physical activity) on all days of the week; TV viewing = television viewing; tertiary family education level = at least one parent has a high school degree; migration background = at least one parent was born abroad or the child was spoken to in foreign language during the first three years of life

*significant difference between children with short/medium and long sleep duration $(p<0.05)$

\section{Sleep duration, physical activity and television viewing}

On average, children slept 9:58 h $( \pm 0: 29)$ per night (ranging from $8 \mathrm{~h} 44 \mathrm{~min}$ to $12 \mathrm{~h} 2 \mathrm{~min})$, with a significant difference between younger and older children $(t=5.72$, $p<0.001)$ but no gender difference. Children of 6 years and younger (average age: $6.09( \pm 0.17)$ years) for example slept on average 10:05 $( \pm 0.33$ ) hours, whereas children of 8 years and older (average age: $8.03( \pm 0.37)$ years) slept 9:45 ( \pm 0:27) hours. Also, children with migration background slept significantly less than children without migration background (9:46 $( \pm 0: 23)$ hours vs. 10:05 ( \pm 0:30) hours, respectively; $\mathrm{t}=4.85, p<0.001$ ).

The participating children spent on average 2:13 ( \pm 0:58) hours per day in MVPA, with a significant gender difference (2:18 ( \pm 0:59) hours for boys vs. 2:04 ( \pm 0:55) hours for girls $\mathrm{t}=9.75, p<0.001)$. Also children spending less than $2 \mathrm{~h}$ in MVPA per day (median; average: 1:28 ( \pm 0.02) hours) slept with 10:02 ( \pm 0:02) hours significantly more than their more active counterparts (average: $2: 58( \pm 0.03)$ hours $)$ with 9:55 $( \pm 0: 28)$ hours $(t=2.20$, $p<0.028)$.

However, merely half of the children (48.7\%) reached the physical activity guideline of $60 \mathrm{~min}$ of MVPA daily. Again, boys achieved this goal significantly more often than girls $(68.7 \%$ vs. $29.2 \%$, respectively; $\mathrm{t}=7.46, p<$ 0.001). Age, migration background or parental education level were not associated with reaching the physical activity guideline, neither was sleep duration (10:01 ( \pm $0: 30$ ) hours for children not reaching the guideline vs.
9:55 ( \pm 0:27) hours for children reaching the guideline); age however was associated with daily MVPA $(\mathrm{t}=-3.05$, $p<0.003)$.

Children spent on average between 45 and 60 min per day watching television, with a significant difference between children from families with migration background and low parental education levels $(\mathrm{t}=-3.54, p<$ 0.001 and $\mathrm{t}=5.02, p<0.001$, respectively). Comparing children who watched television for one hour or more with those who watched television for less than one hour, a significant difference in sleep duration could be observed with children watching more television sleeping less (10:01 ( \pm 0:39) hours vs. 9:50 ( \pm 0:28) hours, $\mathrm{t}=$ $2.22, p<0.027)$.

\section{Associations of BMI z-scores, physical activity and television viewing with sleep duration}

The results from the linear mixed-effects regression models show significant associations between age and migration background with sleep duration (in hours per night) for the total sample and girls, with younger children and those without a migration background sleeping more (see Table 2) and significant associations between age, parental education level and migration background with sleep duration (in hours per night) for boys (see Table 2).

Weight status showed no significant difference in sleep duration with overweight and/or obese children sleeping on average 9:51 ( \pm 0:28) hours whereas normal weight 
Table 2 Associations with daily sleep duration (in hours) displayed for all children, boys and girls (results from linear mixed-effects regression models)

\begin{tabular}{|c|c|c|c|c|c|c|}
\hline & \multicolumn{2}{|l|}{ Total sample } & \multicolumn{2}{|l|}{ Boys } & \multicolumn{2}{|l|}{ Girls } \\
\hline & Estimate [Cl95\%] & $p$ & Estimate [CI95\%] & $p$ & Estimate [Cl95\%] & $p$ \\
\hline Daily MVPA [hours] & $-0.01[-0.01 ; 0.01]$ & 0.423 & $0.01[-0.01 ; 0.01]$ & 0.883 & $-0.01[-0.01 ; 0.01]$ & 0.157 \\
\hline TV viewing of more than $1 \mathrm{~h} /$ day [no vs. yes] & $0.07[-0.10 ; 0.24]$ & 0.423 & $0.09[-0.15 ; 0.34]$ & 0.447 & $0.06[-0.18 ; 0.31]$ & 0.603 \\
\hline BMI z-score $[-]$ & $0.01[-0.05 ; 0.07]$ & 0.726 & $-0.02[-0.10 ; 0.06]$ & 0.599 & $0.04[-0.04 ; 0.13]$ & 0.320 \\
\hline Age [years] & $-0.23[-0.32 ;-0.13]$ & 0.001 & $-0.23[-0.37 ;-0.08]$ & 0.002 & $-0.24[-0.37 ;-0.11]$ & 0.001 \\
\hline Gender [female vs. male] & $-0.10[-0.23 ; 0.04]$ & 0.151 & N/A & N/A & N/A & N/A \\
\hline Tertiary parental education level [no vs. yes] & $0.08[-0.05 ; 0.19]$ & 0.220 & $0.20[0.03 ; 0.37]$ & 0.021 & $-0.06[-0.23 ; 0.11]$ & 0.501 \\
\hline Migration background [no vs. yes] & $0.32[0.19 ; 0.46]$ & 0.001 & $0.23[0.02 ; 0.44]$ & 0.035 & $0.37[0.20 ; 0.55]$ & 0.001 \\
\hline
\end{tabular}

$\mathrm{Cl}$ confidence interval, Daily MVPA = minutes of MVPA (moderate to vigorous physical activity) per day; TV viewing = television viewing; gender = male; tertiary family education level = at least one parent has a high school degree; migration background = at least one parent was born abroad or the child was spoken to in foreign language during the first three years of life

Bold = significant correlates $(p<0.05)$

children slept 8 min longer (9:59 ( \pm 0:30) hours). Also, a decrease of one minute of daily MVPA was associated with longer sleep duration, as was an increase of 0.01 points in BMI z-score (see Table 2), but none of those associations (BMI z-scores, physical activity (in hours per day) or television viewing) were statistically significant.

In the logistic regression analysis a sleep duration of $10 \mathrm{~h}$ and $8 \mathrm{~min}$ or more was associated (not significantly) with lower BMI percentiles $\mathrm{z}$-scores $(0.25 \pm 1.11$ vs. 0.03 \pm 1.10 , respectively; $\mathrm{F}=0.93, p<0.13$ ), and significantly associated with lower daily television viewing (OR 0.45 $[0.27 ; 0.76], p \leq 0.01)$ as well as negatively associated with reaching the physical activity guideline (OR 0.60 $[0.36 ; 0.99], p<0.05)$. However, if controlling for age, gender, parental education level and migration background, reaching the physical activity guideline stayed no longer significantly associated with a tertiary sleep level (see Table 3).

For the total sample, a long sleep duration was significantly positively associated with daily television viewing of less than one hour (OR 0.44 [0.24;0.80]), young age (OR $0.38[0.21 ; 0.67])$, a high parental education level
(OR $0.52[0.27 ; 0.99]$ ) and the lack of migration background (OR 0.21 [0.10;0.48]; see Table 3).

If analysed separately for boys and girls, only having a migration background was significantly associated with a tertiary sleep level for both genders (OR 0.12 [0.02;0.56] and OR 0.27 [0.10;0.72], respectively). For boys, neither age nor television viewing stayed significantly associated with a long sleep duration, but parental education level still showed a significant association (OR 0.25 $[0.09 ; 0.72])$. For girls on the other hand, young age and little television viewing were significant positive correlates of a tertiary sleep level (OR $0.27[0.12 ; 0.60]$ and OR $0.34[0.15 ; 0.77]$, respectively), however parental education lost its significance (see Table 3). Originally planned clustering for schools was neglected as no associations were found.

\section{Discussion}

This study investigated associations of objectively assessed night-time sleep duration with daily MVPA, reaching the physical activity guideline of $60 \mathrm{~min}$ of MVPA per day, daily television viewing and body composition in the form of BMI z-scores in German primary

Table 3 Odds Ratios for tertiary sleep level (results from multiple logistic regression models)

\begin{tabular}{|c|c|c|c|c|c|c|}
\hline & \multicolumn{2}{|l|}{ Total sample } & \multicolumn{2}{|l|}{ Boys } & \multicolumn{2}{|l|}{ Girls } \\
\hline & OR [C195\%] & $p$ & OR [C195\%] & $p$ & OR [C195\%] & $p$ \\
\hline MVPA Guideline reached & $0.68[0.39 ; 1.20]$ & 0.182 & $0.99[0.39 ; 2.51]$ & 0.981 & $0.79[0.33 ; 1.89]$ & 0.598 \\
\hline TV viewing of more than $1 \mathrm{~h} /$ day & $0.44[0.24 ; 0.80]$ & 0.007 & $0.46[0.17 ; 1.24]$ & 0.123 & $0.34[0.15 ; 0.77]$ & 0.009 \\
\hline Age & $0.38[0.21 ; 0.67]$ & 0.001 & $0.44[0.18 ; 1.05]$ & 0.064 & $0.27[0.12 ; 0.60]$ & 0.001 \\
\hline Tertiary parental education level & $0.52[0.27 ; 0.99]$ & 0.046 & $0.25[0.09 ; 0.72]$ & 0.010 & $1.05[0.44 ; 2.50]$ & 0.910 \\
\hline Migration background & $0.21[0.10 ; 0.48]$ & 0.000 & $0.12[0.02 ; 0.56]$ & 0.007 & $0.27[0.10 ; 0.72]$ & 0.009 \\
\hline
\end{tabular}

OR Odds Ratio, $\mathrm{Cl}$ confidence interval; MVPA guideline reached = a minimum of 60 min of MVPA (moderate to vigorous physical activity) on all days of the week; TV viewing = television viewing; tertiary family education level = at least one parent has a high school degree; migration background = at least one parent was born abroad or the child was spoken to in foreign language during the first three years of life

Bold $=$ significant correlates $(p<0.05)$ 
school children. On average, children slept short of $10 \mathrm{~h}$, with younger children and those without migration background sleeping significantly more than older children and those with migration background. This could also be confirmed by the results from the linear regression models investigating associations between daily physical activity, television viewing and body composition.

In order to investigate differences in children with long and short sleep duration, three sleep levels were formed. Children with long sleep duration, compared to those with short and medium sleep duration, were grouped in the tertiary sleep level, which was classified as a sleep duration of $10 \mathrm{~h}$ and $8 \mathrm{~min}$ or more per night. This was - if individually observed - significantly negatively associated with reaching the physical activity guideline of $60 \mathrm{~min}$ of MVPA as well as positively associated with daily television viewing of less than one hour. Children's BMI z-scores based on Cole et al. [39] showed no association with sleep duration, BMI percentiles if classified based on German reference data [49] on the other hand were significantly associated with the tertiary sleep level (data not shown).

Several previous studies have found that sleep duration may play a key role in the development of overweight and obesity [22, 25, 50]. In school-aged children for instance, the risk of being overweight or obese in children who had less than $10 \mathrm{~h}$ of sleep on non-school days was far greater than in those who slept more than $10 \mathrm{~h}$ per night [28]. In this study however, neither normal weight nor overweight children averaged $10 \mathrm{~h}$ of sleep. Still, short sleep duration during childhood was not only associated with present overweight but also with increased body weight in later childhood and adolescence $[25,51]$ as well as higher BMI values and diabetes risk markers [52]. It could be shown that children with too little sleep at 5 to 6 years of age are more likely to be overweight at 15 years [25] and also children with a short sleep duration at 4 to 5 years showed significantly higher BMI values at 8 and 9 years of age [51]. The latter however, was partially mediated by increased television viewing at 6 to 7 years of age [51].

Apart from such behavioural aspects as television viewing, the suggested reasons for the relationship of sleep and weight status in children vary. Possible approaches assume that sleep duration may be independently associated with children's metabolic body size phenotype [53] but also that too little sleep can lead either to an increased energy intake or to a reduced metabolic function [22, 54]. Both could have a hormonal explanation, since short sleep duration is associated with increased ghrelin levels (which are known to increase one's appetite) and a reduced release of the appetite-inhibiting hormone leptin [55-58]. Further has been shown, that sleep deficiency is associated with a stimulation of certain regions in the brain which are sensitive to food stimuli, which points to the assumption that too little sleep might lead to obesity through the selection of high caloric food [54].

Explanations such as the above mentioned are supported by the fact that children who sleep less than $10 \mathrm{~h}$ per night consume soft drinks more frequently, eat vegetable less often and consume greater amounts of fried food than children whose sleep duration is longer than $10 \mathrm{~h}$ [28, 59]. Further, a long-term study covering a period of 32 years showed that lack of sleep is associated to an increased BMI independently of other behavioural aspects such as media use and socioeconomic status [60].

Behavioural factors however have also been examined in this study; daily television viewing of one hour or less was not associated with children's sleep duration when analysed in linear regression models but showed significant associations with the tertiary sleep level if analysed in logistic regression models. This is also highlighted by a recent review investigating associations between screen media use and sleep in children and adolescents 5- to 17 years old from different regions around the world [30]. In over $90 \%$ of the included studies, more time with screen media was associated with delayed bedtimes and shorter sleep duration among children and adolescents [30]. Among studies associating television viewing with sleep timing and/or quality, over $75 \%$ found significant relationships between television watching and too little sleep [30].

This is consistent with most previous research, showing that children with longer periods of television viewing also sleep for shorter periods of time [61-64]. Often this has been attributed to delayed bedtime [65, 66] (which has not been considered in this study) but also to physiological suppression of the sleep-promoting hormone melatonin through the bright light of screens [67]. However, even longitudinal investigations of two-, four, six- and nine-year-olds show, that children with more television use ( $1.5 \mathrm{~h}$ per day or more) at baseline have shorter sleep duration, as well as having a reduction in sleep duration at follow-up with inverse associations of changes in sleep duration [62].

This sample showed comparably low television viewing levels with children spending on average between 45 and $60 \mathrm{~min}$ per day watching television. If watching television for one hour or more per day children slept on average $11 \mathrm{~min}$ less than those watching less than one hour per day. If they came from families with migration background and low parental education levels television viewing was significantly higher. Once controlled for 
those factors, for boys, television viewing no longer stayed significantly associated with long sleep duration. For girls on the other hand, low television use was still significantly positively correlated to a tertiary sleep level if controlling for socioeconomic variables.

Similarly, despite the initial significant association between children's short sleep duration and them reaching the physical activity guideline of $60 \mathrm{~min}$ of MVPA on every day of the week [17] in the logistic regression model, once controlled for age, gender, parental education level and migration background, reaching the physical activity guideline stayed no longer significantly associated with a tertiary sleep level. Also, analysing daily MVPA in hours, independent of reaching the recommended 60 min MVPA per day, in linear regressions no association with sleep duration could be found. Yet, there was a difference of 7 min found when comparing more active children with less active children, which also made a difference whether children slept more or less than $10 \mathrm{~h}$. Therefore, children who spent less time in MVPA slept more (than $10 \mathrm{~h}$ ). Similarly, previous research has shown inconsistent finding regarding children's physical activity levels. In a recent subjectively assessed sample of 13,000 primary school children in China, children with a sleep duration of $10 \mathrm{~h}$ or less were more likely to engage in more moderate and low level physical activity, compared to children sleeping 10 $\mathrm{h}$ or more per night [28]. Vigorous physical activity however, was not associated with sleep duration and no physical activity (duration or frequency) was associated with the amount of sleep on weekends [28]. Although other studies have shown relationships between longer sleep duration and increased physical activity $[68,69]$, a study assessing children slightly younger than the ones in this sample, found that - objectively assessed - the most active children slept 1:02 and 1:40 h less at night compared with the least active children at 5 and 7 years, respectively [70]. Also, a sleep duration of an additional hour in 10- to 12-year-olds has been associated with 20 min less of MVPA during the day [32]; whereas other studies show no relationship between sleep duration and physical activity levels on the following day [24].

Since most studies assess cross-sectional non-experimental data on sleep and physical activity with no meaningful statement on whether physical activity affects sleeping patterns or the other way round, recent research analysed bi-directional associations between sleep duration and MVPA [71]. Lin and colleagues [71] were able to show that if children's sleep duration was increased by one hour, their time spent in MVPA increased by less than one minute. Further, if MVPA was to predict sleep duration, MVPA was significantly associated with sleep duration; for each one hour increase in MVPA, sleep duration increased by six minutes [71].
In order to investigate and clarify this question further, more longitudinal experimental research with objective measurement methods are needed. However, until then, this research adds valuable insights in associations of children's sleep duration and behavioural as well as physical factors. In spite of this, these findings should be interpreted with caution since there are some limitations that need to be considered. Since physical activity was estimated on the basis of energy expenditure it could have led to misinterpretations of some children's intensities and therefore the reported results. Further, the used device recorded children's activity four times per minute, which may have been not often enough in order to accurately capture every activity. Further, information on television watching was provided by parents in a questionnaire, which is subject to a reporting bias. Moreover, a potential selection bias cannot be ruled out since children, parents and schools participated on a voluntary basis. Furthermore should be noted that the sample - although from a quite widely spread area - is not representative, mainly due to its low overweight and obesity prevalence. A further limitation is the cross-sectional design of this study which does not allow for causal interpretation and the study is relatively small and therefore underpowered, which limits observing potentially significant associations. Additionally, when analysed with logistic regression models, six children ( $2 \%$ of the cohort) who slept more than the recommended 9 to $11 \mathrm{~h}$ per night, we included in the highest sleep level, which was not further considered and could also contribute to potential health issues. Despite these limitations, the comprehensive assessment of children's weight status as well as the consideration of a multiplicity of independent factors should be considered a strength of this study. Further strengths of this study are the objective measurement of body composition and the individual calculation of objectively assessed sleep duration and physical activity which allow a certain reliability of data.

\section{Conclusions}

The here investigated associations of objectively assessed night-time sleep duration with reaching the physical activity guideline of $60 \mathrm{~min}$ of MVPA per day and daily television viewing in German primary school children, showed that - although not associated when analysed in linear regression models both are independently associated with a tertiary sleep level of $10 \mathrm{~h}$ and $8 \mathrm{~min}$ per night or more. This therefore adds to previously primarily subjectively assessed associations of sleep and risk factors for obesity (related behaviours) with a detailed insight based on 
objective data. However, more longitudinal and experimental research is needed in order to provide reliable data in order to enable decision making of public health stakeholders, policymakers and practitioners. Interventions trying to decrease children's BMI and television viewing should also aim at extending children's night-time sleep and inform parents about the importance of sufficient sleep during childhood in order to pursue a holistic health promotion.

\section{Abbreviations}

BMI z-scores: body mass index z-scores; BMl: body mass index; MVPA: moderate to vigorous physical activity; WHO: World Health Organisation

\section{Acknowledgements}

The authors acknowledge and thank all members of the "Join the Healthy Boat" team including cooperating institutes, participating schools, teachers, parents and children.

\section{Funding}

The programme as well as the study was financed by the BadenWürttemberg Stiftung (Grant No. BWS_1.479.00_2009), which had no influence on either data or manuscript.

\section{Availability of data and materials}

The datasets used and/or analysed during the current study are available from the corresponding author on reasonable request.

\section{Authors' contributions}

SKo designed and carried out the study, analysed the data, performed the statistical analysis and drafted the manuscript. OW designed and carried out the study and revised the manuscript. JD designed the study, prepared the data, supported the statistical analysis and revised the manuscript. SKe designed and carried out the study, analysed the data and revised the manuscript. JMS designed the study and revised the manuscript. All authors read and approved the final manuscript.

\section{Ethics approval and consent to participate}

The study was approved and consented by the University of Ulm's ethics committee (No. 126/10), conducted in accordance with the declaration of Helsinki and is registered at the German Clinical Trials Register (DRKS-ID: DRKS00000494). Parents provided their written informed consent to take part in the study and a separate consent for their children to wear a multi-sensor device assessing sleep and physical activity objectively for six consecutive days; children gave their verbal assent on the day of assessment.

\section{Consent for publication}

Not applicable.

\section{Competing interests}

The authors declare that they have no competing interests.

\section{Publisher's Note}

Springer Nature remains neutral with regard to jurisdictional claims in published maps and institutional affiliations.

\section{Author details}

'Division of Sports and Rehabilitation Medicine, Department of Internal Medicine II, Ulm University Medical Centre, Frauensteige 6, Haus 58/33, D-89075 Ulm, Germany. ${ }^{2}$ Institute of Epidemiology and Medical Biometry, Ulm University, Schwabstr. 13, D-89075 Ulm, Germany. ${ }^{3}$ Division of Sports and Rehabilitation Medicine, Department of Internal Medicine II, Ulm University Medical Centre, Leimgrubenweg 14, D-89075 Ulm, Germany.
Received: 8 May 2018 Accepted: 7 February 2019

Published online: 11 February 2019

\section{References}

1. Seidell JC, Halberstadt J. The global burden of obesity and the challenges of prevention. Ann Nutr Metab. 2015;66(2):7-12.

2. Kuciene $R$, Dulskiene $V$, Medzioniene J. Association of neck circumference and high blood pressure in children and adolescents: a case-control study. BMC Pediatr. 2015:15(1):127-36.

3. Grigorakis DA, Georgoulis M, Psarra G, Tambalis KD, Panagiotakos DB, Sidossis LS. Prevalence and lifestyle determinants of central obesity in children. Eur J Nutr. 2016;55(5):1923-31.

4. Cataldo R, Huang J, Calixte R, Wong AT, Bianchi-Hayes J, Pati S. Effects of overweight and obesity on motor and mental development in infants and toddlers. Pediatr Obes. 2016;11(5):389-96.

5. Dev DA, McBride BA, Fiese BH, Jones BL, Cho H. Risk factors for overweight/ obesity in preschool children: an ecological approach. Child Obes. 2013;9(5): 399-408.

6. Ng M, Fleming T, Robinson M, Thomson B, Graetz N, Margono C, et al. Global, regional, and national prevalence of overweight and obesity in children and adults during 1980-2013. A systematic analysis for the Global Burden of Disease Study 2013. Lancet. 2014:384(9945):766-81.

7. $\mathrm{WHO}$ - World Health Organization. Global status report on noncommunicable diseases, vol. 2014. Geneva: World Health Organization; 2014

8. WHO - World Health Organization. Overweight and obesity - Fact sheet. 2018. http://www.who.int/mediacentre/factsheets/fs311/en/. Accessed 8 Feb 2019.

9. Savage JS, Orlet Fisher J, Birch LL. Parental influence on eating behavior: conception to adolescence. J Law Med Ethics. 2007:35(1):22-34.

10. Zecevic CA, Tremblay L, Lovsin T, Michel L. Parental influence on Young Children's physical activity. Int J Pediatr. 2010:2010:468526.

11. WHO - World Health Organization. Draft final report of the commission on ending childhood obesity. Geneva: WHO Document Production Services; 2015.

12. Must A, Barish EE, Bandini LG. Modifiable risk factors in relation to changes in BMI and fatness: what have we learned from prospective studies of school-aged children? Int J Obes. 2009;33:705-15.

13. Wheaton $\mathrm{N}$, Millar L, Allender S, Nichols M. The stability of weight status through the early to middle childhood years in Australia: a longitudinal study. BMJ Open. 2015;5(4):1-9.

14. Sahoo K, Sahoo B, Choudhury AK, Sofi NY, Kumar R, Bhadoria Ajeet S. Childhood obesity: causes and consequences. J Family Med Prim Care. 2015:4(2):187-92

15. Andersen LB, Harro M, Sardinha LB, Froberg K, Ekelund U, Brage S, et al. Physical activity and clustered cardiovascular risk in children: a crosssectional study (the European youth heart study). Lancet. 2006;368:299304.

16. Janssen I, LeBlanc AG. Systematic review of the health benefits of physical activity and fitness in school-aged children and youth. Int J Behav Nutr Phys Act. 2010;7:40.

17. WHO - World Health Organization. Global recommendations on physical activity for health. Geneva: World Health Organization; 2010.

18. Guinhouya BC, Samouda H, de Beaufort C. Level of physical activity among children and adolescents in Europe: a review of physical activity assessed objectively by accelerometry. Pub Health. 2013;127:301-11.

19. Song M, Carroll DD, Fulton JE. Meeting the 2008 physical activity guidelines for Americans among U.S. youth. Am J Prev Med. 2013;44(3):216-22

20. The Health and Social Care Information Centre. Health Survey for England 2008: Physical activity and fitness. Leeds: NHS Information Centre; 2009.

21. Krug S, Jekauc D, Poethko-Müller C, Woll A, Schlaud M. Zum Zusammenhang zwischen körperlicher Aktivität und Gesundheit bei Kindern und Jugendlichen. Ergebnisse des Kinder- und Jugendgesundheitssurveys (KiGGS) und des Motorik Moduls (MoMo) [Relationship between physical activity and health in children and adolescents Results of the German Health Interview and Examination Survey for Children and Adolescents (KiGGS) and the "Motorik-Modul" (MoMo)] Bundesgesundheitsbl Gesundheitsforsch Gesundheitsschutz 2012;55:111-20

22. Chaput JP, Lambert M, Gray-Donald K, McGrath JJ, Tremblay MS, O'Loughlin J, Tremblay A. Short sleep duration is inde-pendently associated with overweight and obesity in Quebec children. Can J Public Health. 2011; 102(5):369-74. 
23. Börnhorst C, Hense S, Ahrens W, Hebestreit A, Reisch L, Barba G, et al. From sleep duration to childhood obesity--what are the path-ways? Eur J Pediatr. 2012;171(7):1029-38

24. Ekstedt M, Nyberg G, Ingre M, Ekblom Ö, Marcus C. Sleep, physical activity and BMI in six to ten-year-old children measured by accelerometry: a crosssectional study. Int J Behav Nutr Phys Act 2013;10(82):1-10.

25. Bonuck K, Chervin RD, Howe LD. Sleep-disordered breath-ing, sleep duration, and childhood overweight: a longitudinal cohort study. J Pediatr. 2015;166(3):632-9.

26. Hense S, Pohlabeln H, de Henauw S, Eiben G, Molnar D, Moreno LA, et al. Sleep duration and overweight in European children: is the association modified by geographic region? Sleep. 2011;34(7):885-90.

27. Hirshkowitz M, Whiton K, Albert SM, Alessi C, Bruni O, DonCarlos L, et al. National Sleep Foundation's sleep time duration recommendations. Methodology and results summary. Sleep Health. 2015;1(1):40-3.

28. Zhang J, Zhang YT, Jiang YR, Sun WQ, Zhu Q, Ip P, Zhang DL, et al. Effect of sleep duration, diet, and physical activity on obesity and overweight elementary school students in Shanghai. J Sch Health. 2018;88:112-21.

29. Pulido-Arjona L, Correa-Bautista JE, Agostinis-Sobrinho C, Mota J, Santos R, Correa-Rodríguez M, et al. Role of sleep duration and sleep-related problems in the metabolic syndrome among children and adolescents. Ital J Pediatr. 2018;44:9

30. Hale L, Guan S. Screen time and sleep among school-aged children and adolescents: a systematic literature review. Sleep Med Rev 2015;21:e50-8.

31. Hart CN, Carskadon MA, Considine RV, Fava JL, Lawton J, Raynor HA, et al. Changes in children's sleep duration on food intake, weight, and leptin. Pediatr. 2013;132:e1473-80.

32. Sorić M, Starc H, Borer KT, Jurak G, Kovač M, Strel J, et al. Associations of objectively assessed sleep and physical activity in 11-year old children. Ann Hum Biol. 2015:42(1):31-7.

33. Pesonen AK, Sjöstén NM, Matthews KA, Heinonen K, Martikainen S, Kajantie E, et al. Temporal associations between daytime physical activity and sleep in children. PLoS One 2011. 6(8):e22958.

34. Vincent GE, Barnett LM, Lubans DR, Salmon J, Timperio A, Ridgers ND. Temporal and bidirectional associations between physical activity and sleep in primary school-aged children. Appl Physiol Nutr Metab 2017. 42(3):238-42

35. Lin Y, Borghese MM, Janssen I. Bi-directional association between sleep and outdoor active play among 10-13 year olds. BMC Pub Health. 2018;18:224-31.

36. Bauer K, Blunden S. How accurate is subjective reporting of childhood sleep patterns? A review of the literature and implications for practice. Curr Pediatr Rev. 2008;4(2):132-42.

37. Kobel S, Wirt T, Schreiber A, Kesztyüs D, Kettner S, Erkelenz N, et al. Intervention effects of a school-based health promotion Programme on obesity related Behavioural outcomes. J Obes. 2014:1-8.

38. Dreyhaupt J, Koch B, Wirt T, Schreiber A, Brandstetter S, Kesztyüs D, et al. Evaluation of a health promotion program in children: study protocol and design of the cluster-randomized Baden-Württemberg primary school study. BMC Pub Health. 2012;12(157):1-12

39. Cole TJ, Bellizzi MC, Flegal KM, Dietz WH. Establishing a standard definition for child overweight and obesity worldwide: international survey. BMJ. 2000; 320:1240-3.

40. Corder K, Brage S, Wareham NJ, Ekelund U. Comparison of PAEE from combined and separate heart rate and movement models in children. Med Sci Sports Exerc. 2005;37:1761-7.

41. Sadeh A. The role and validity of actigraphy in sleep medicine: an update practice points, research agenda. Sleep Med Rev. 2011;15(4):259-67.

42. Wearables HHC. Die Bedeutung der neuen Technologie für die Sportmedizin [Wearables - the meaning of a new technology for sports medicine]. Dtsch Z Sportmed. 2016;67:285-6.

43. Kettner S, Kobel S, Fischbach N, Drenowatz C, Dreyhaupt J, Wirt T, et al. Objectively determined physical activity levels of primary school children in south-West Germany. BMC Pub Health. 2013;13:895-904.

44. Trost SG, Pate RR, Freedson PS, Sallis JF, Taylor WC. Using objective physical activity measures with youth: how many days of monitoring are needed? Med Sci Sports Exerc. 2000;32:426-31

45. Sack RL, Auckley D, Auger R, Carskadon MA, Wright KP Jr, Vitiello MV, et al. Circadian rhythm sleep disorders: part II, advanced sleep phase disorder delayed sleep phase disorder, free-running disorder, and irregular sleepwake rhythm. Sleep. 2007;30(11):1484-501.

46. Pate RR, Pratt M, Blair SN, Haskell WL, Macera CA, Bouchard C, et al. Physical activity and public health: a recommendation from the Centers for Disease
Control and Prevention and the American College of Sports Medicine. JAMA. 1995:273:402-7.

47. Kurth BM, Schaffrath Rosario A. Übergewicht und Adipositas bei Kindern und Jugendlichen in Deutschland [Overweight and obesity in children and adolescents in Germany]. Bundesgesundheitsbl Gesundheitsforsch Gesundheitsschutz. 2010:53(7):643-52.

48. Rütten A, Pfeiffer K (Eds.). Nationale Empfehlungen für Bewegung und Bewegungsförderung. [national recommendations for physical activity and physical activity promotion]. Erlangen-Nürnberg: FAU; 2016.

49. Kromeyer-Hauschild K, Wabitsch M, Kunze D, Geller F, Geiß HC, Hesse V, et al. Perzentile für den Body-mass-Index für das Kindes- und Jugendalter unter Heranziehung verschiedener deutscher Stichproben Percentiles of body mass index in children and adolescents evaluated from different regional German studies]. Monatsschr Kinderheilkd. 2001; 149(8):807-18.

50. Moraleda-Cibrián M, O'Brien LM. Sleep duration and body mass index in children and adolescents with and without obstructive sleep apnea. Sleep Breath. 2014;18:555-61

51. Magee C, Caputi P, Iverson D. Lack of sleep could increase obesity in children and too much television could be partly to blame. Acta Paediatr. 2014;103:e27-31.

52. Rudnicka AR, Nightingale CM, Donin AS, Sattar N, Cook DG, Whincup PH, Owen CG. Sleep duration and risk of type 2 diabetes. Pediatrics. 2017;140(3): e20170338

53. Lim HH. Sleep duration independently influences metabolic body size phenotype in children and adolescents: a population-based study. Sleep Med. 2018;42:47-52.

54. Copinschi G, Leproult R, Spiegel K. The important role of sleep in metabolism. Front Horm Res. 2014;42:59-72.

55. Spiegel K, Tasali E, Penev P, Van CE. Brief communication: sleep curtailment in healthy young men is associated with decreased leptin levels, elevated ghrelin levels, and increased hunger and appetite. Ann Intern Med. 2004; 141:846-50.

56. Spiegel K, Leproult R, L'hermite-Baleriaux M, Copinschi G, Penev PD, Van Cauter E. Leptin levels are dependent on sleep duration: relationships with sympathovagal balance, carbohydrate regulation, cortisol, and thyrotropin. J Clin Endocrinol Metab. 2004;89:5762-71.

57. Chaput JP, Despres JP, Bouchard C, Tremblay A. Short sleep duration is associated with reduced leptin levels and increased adiposity: results from the Quebec family study. Obesity. 2007;15:253-61.

58. Taheri S, Lin L, Austin D, Young T, Mignot E. Short sleep duration is associated with reduced leptin, elevated ghrelin, and increased body mass index. PLOS Med. 2004:1:e62.

59. Franckle RL, Falbe J, Gortmaker S, Ganter C, Taveras EM, Land T, et al. Insufficient sleep among elementary and middle school students is linked with elevated soda consumption and other unhealthy dietary behaviours. Prev Med. 2015;74:36-41.

60. Landhuis CE, Poulton R, Welch D, Hancox RJ. Childhood sleep time and long-term risk for obesity. A 32-year prospective birth cohort study. Pediatrics. 2008;122(5):955-60.

61. Cain N, Gradisar M. Electronic media use and sleep in school-aged children and adolescents. A review. Sleep Med. 2010;11(8):735-42.

62. Marinelli M, Sunyer J, Alvarez-Pedrerol M, Iñiguez C, Torrent M, Vioque J, et al. Hours of television viewing and sleep duration in children. A multicentre birth cohort study. JAMA Pediatr. 2014;168(5):458-64.

63. Falbe J, Davison KK, Franckle RL, Gantner C, Gortmaker SL, Smith L, et al. Sleep duration, restfulness, and screens in the sleep environment. Pediatrics. 2015;135(2):e367-75.

64. LeBourgeois MK, Hale L, Chang AM, Akacem LD, Montgomery-Downs HE, Buxton OM. Digital media and sleep in childhood and adolescence. Pediatrics. 2017;140(2):92-6.

65. Abe T, Hagihara A, Nobutomo K. Sleep patterns and impulse control among Japanese junior high school students. J Adolesc. 2010;33(5):633-41.

66. Adam EK, Snell EK, Pendry P. Sleep timing and quantity in ecological and family context: a nationally representative time-diary study. J Fam Psychol. 2007;21(1):4

67. Higuchi S, Motohashi Y, Liu Y, Maeda A. Effects of playing a computer game using a bright display on presleep physiological variables, sleep latency, slow wave sleep and REM sleep. J Sleep Res. 2005;14(3):267-73.

68. Kjeldsen JS, Hjorth MF, Andersen R, Michaelsen KF, Tetens I, Astrup A, et al. Short sleep duration and large variability in sleep duration are independently 
associated with dietary risk factors for obesity in Danish school children. Int J Obes. 2014;38:32-9.

69. Khan MK, Chu YL, Kirk SF, Veugelers PJ. Are sleep duration and sleep quality associated with diet quality, physical activity, and body weight status? A population-based study of Canadian children. Can J Public Health. 2015;106: e277-82.

70. Williams SM, Farmer VL, Taylor BJ, Taylor RW. Do more active children sleep more? A repeated cross-sectional analysis using accelerometry. PLoS One. 2014;9(4):e93117.

71. Lin Y, Tremblay MS, Katzmarzyk PT, Fogelholm M, Hu G, Lambert EV, et al. Temporal and bi-directional associations between sleep duration and physical activity/sedentary time in children: an international comparison. Prev Med 2017; [Epub ahead of print].

Ready to submit your research? Choose BMC and benefit from:

- fast, convenient online submission

- thorough peer review by experienced researchers in your field

- rapid publication on acceptance

- support for research data, including large and complex data types

- gold Open Access which fosters wider collaboration and increased citations

- maximum visibility for your research: over $100 \mathrm{M}$ website views per year

At BMC, research is always in progress.

Learn more biomedcentral.com/submissions 